\title{
Experiencia de enseñanza usando metodologías activas, $y$ tecnologías de información y comunicación en estudiantes de medicina del ciclo clínico
}

\author{
Mauricio Castillo-Montes y Muriel Ramírez-Santana \\ Departamento de Salud Pública, Facultad de Medicina, Universidad Católica del Norte, Larrondo 1281, Coquimbo - \\ Chile. (correo-e: mcmontes@ucn.cl; mramirezs@ucn.cl)
}

Recibido Oct. 4, 2019; Aceptado Nov. 19, 2019; Versión final Ene. 16, 2020, Publicado Jun. 2020

\begin{abstract}
Resumen
El objetivo del estudio fue evaluar cambios en rendimiento académico y satisfacción de estudiantes de medicina en la asignatura salud ambiental y ocupacional, al incorporar mejoras docentes, consistentes en metodologías activas y en tecnologías de información y comunicación (TIC). Se efectuó un estudio cuasi experimental retrospectivo utilizando 450 registros de calificaciones y 343 encuestas de percepción divididas en dos periodos: "antes" (2008-2011) y "después" (2012-2016) de implementar las mejoras docentes. La calificación promedio del período tuvo una tendencia ascendente. El periodo antes tuvo casi nula variación lineal y el periodo después tuvo una fuerte tendencia al aumento en las notas. La encuesta de satisfacción presentó diferencias entre ambos períodos, aumentando significativamente en pertinencia curricular y ayudantías. El uso de metodologías activas contribuye a fomentar el aprendizaje activo, mejorando la satisfacción y calificaciones de los estudiantes, especialmente de aquellos con menor desempeño académico.
\end{abstract}

\section{Teaching experience using active methodologies and information and communication technologies in medical students of the clinical cycle}

\begin{abstract}
The objective of the study was to evaluate changes in academic performance and satisfaction of medical students in the environmental and occupational health course. This was performed by incorporating educational improvements consisting of active methodologies and information and communication technologies. A quasi-experimental retrospective study was performed on 450 grade records and 343 perception surveys that were divided in two periods: 1) "before" implementing teaching improvements (20082011) and 2) "after" implementing teaching improvements (2012-2016). The average period score showed an upward trend. The "before" period showed no linear variation but the "after" period showed a strong tendency to increase in the qualifications observed. The satisfaction survey presented differences between both periods, increasing significantly in curricular relevance and assistantships. The use of active methodologies contributes to promoting active learning and approval and qualifications of students, especially for lower academic performance students.
\end{abstract}

Keywords: medical education; academic performance; student satisfaction; teaching methods; information technologies 


\section{INTRODUCCIÓN}

En los últimos años, las facultades de medicina de las universidades chilenas han desarrollado reformas curriculares, cambios en los procesos educativos y en las prácticas de la docencia, con el propósito de mejorar el proceso enseñanza - aprendizaje (EA) de los futuros profesionales. Dichas transformaciones han requerido modificaciones en la gestión organizacional y en la formación docente. Principalmente, en Chile, estos cambios se han producido en universidades complejas; entendidas como aquellas que desarrollan no tan sólo docencia y gestión universitaria, sino también vinculación con el medio, especialmente, investigación básica, investigación aplicada e innovación; generando productividad científica de alto nivel (Ministerio de Educación, 2017). En este tipo de universidades, donde los académicos combinan docencia, investigación y vinculación con el medio (Castillo et al., 2014), y además, para la formación de médicos, ha sido necesaria la adaptación de los perfiles de egreso de los profesionales, hacia una mayor coherencia con las necesidades de salud y sociales del país (Parada et al., 2015).

Un importante rol en educación médica ha jugado la implementación de metodologías activas y participativas de enseñanza, que para su ejecución requieren contextos organizacionales y culturales. El contexto organizacional, se refiere a los planes estratégicos o corporativos por los cuales las universidades especifican sus objetivos en los diferentes ejes, tales como; docencia (a través de sus proyectos educativos institucionales), investigación, vinculación con el medio, gestión institucional, internacionalización, identidad y comunicaciones; el contexto cultural, tiene relación con cómo las instituciones perciben los cambios culturales en todos los ámbitos de la vida social, que en la actualidad se motivan principalmente por el fenómeno de la globalización, y cómo reflexionan críticamente frente a estas nuevas relaciones sociales en su quehacer científico, creativo y cultural. Frente a este escenario, los docentes debieran acceder a perfeccionamiento académico de tipo formal; tanto en las áreas disciplinares como en el ámbito pedagógico. El dominio de estas dos áreas (disciplinar y pedagógica) es relevante para la docencia actual en ciencias de la salud; ya que, siendo competencias diferentes, ninguna está por sobre la otra.

En lo particular, las estrategias de EA en la educación médica, se han visto obligadas a ir evolucionando a través del tiempo, debido a las crecientes demandas para mejorar la atención del paciente y de la comunidad, sin descuidar la responsabilidad social, moral y ética. Por esta razón, los procesos de EA se centran en el estudiante, con una visión coherente y articulada entre los objetivos y la secuencia lógica de los procesos, con el propósito de que el estudiante perciba que, además de la adquisición de conocimientos, también existen muchas formas o entornos de aprendizaje para el logro de resultados. Dicha situación exige cada vez mayores necesidades de perfeccionamiento docente, especialmente en estrategias de EA, métodos didácticos y evaluación. Especial atención se ha puesto en los evidentes efectos positivos de las estrategias pedagógicas orientadas por el modelo constructivista (Castillo et al, 2014; Pérez et al., 2013; McLean et al., 2008; Troncoso et al., 2017).

En este contexto educativo, se desarrollan las Tecnologías de Información y Comunicación (TIC) - entendidas como tecnologías relacionadas con la capacidad para representar y transmitir información - las cuales utilizan sistemas de signos tales como; el lenguaje oral y escrito, imágenes estáticas y en movimiento, símbolos matemáticos, entre otros. Junto a la Internet, han producido importantes cambios en la sociedad actual, lo que se ha denominado la Sociedad de la Información. Tales cambios han afectado a casi todos los ámbitos de las actividades cotidianas, la forma de organización social, comprensión del mundo y transmisión de la información entre personas (Coll y Monereo, 2008).

La UNESCO plantea en sus directrices dirigidas a los actores de educación superior, que los gobiernos deben promover el uso de TIC mediante el suministro sostenido de conectividad y acceso, y orientan a las universidades para asegurar que académicos y estudiantes puedan acceder a estas tecnologías mediante infraestructura, conectividad, softwares y repositorios adecuados, manteniendo permanentemente formación, desarrollo profesional y apoyo en estos sistemas. Consecuente con lo anterior, en las instituciones de educación superior, existe una tendencia creciente para incorporar las TIC en sus programas de gestión, administración y educación, con los propósitos de tener mayor eficiencia y que la preparación de los estudiantes se oriente al mundo actual. Para ello, han adaptado sus enfoques pedagógicos y materiales didácticos, utilizando sistemas de comunicación globales para aumentar en forma vertiginosa la generación y el intercambio del conocimiento (Organización de las Naciones Unidas para la Educación, la Ciencia y la Cultura, 2015).

Las TIC, donde la mediación didáctica aparece conciliada por la tecnología más que un mero soporte, promueven la necesidad de inmediatez, de ver y oír en pocos minutos, lo que produce el efecto de vivir el presente dificultando postergar deseos y decisiones. Sin embargo, los procesos educativos manifiestan la importancia del análisis y reflexión antes que una respuesta apresurada, lo que debe revalorizarse en la actualidad. En educación, las TIC han producido cambios en las formas de pensar, conocer y comunicarse; 
en la forma de pensar, se manifiesta una mayor complejidad, que se ve reflejada en el diálogo con la incertidumbre más que llegar a la verdad absolutamente cierta, por lo tanto, se requiere dotar a los estudiantes de competencias cognitivas y metacognitivas más exigentes; en cuanto a las formas de conocer y gestionar el conocimiento (concepción epistemológica), las TIC promueven el pluralismo representacional, al convivir con múltiples perspectivas, que incluso pueden transformar la realidad o vivir realidades virtuales, lo que debe observarse con cautela cuando se utiliza información de poca fiabilidad; finalmente, en las formas de comunicación, han producido cambios en la velocidad de intercambios de informaciones y representaciones, los que son prácticamente inmediatos (Coll y Monereo, 2008).

De lo anterior, el uso de Tecnologías de Información y Comunicación (TIC) en los procesos de docencia universitaria, han permitido que la distancia física y la asincronía no sean una limitante en la comunicación y el aprendizaje. Sin embargo, exige voluntad de comunicarse y relacionarse; además de una adecuada planificación y organización por parte del docente. El uso de TIC complementa las actividades presenciales y clases magistrales tradicionales, favoreciendo el uso de diversas didácticas (Bautista et al., 2008). Adicionalmente, el uso de TIC como estrategia de EA, fortalece la autonomía del estudiante, permiten al profesor modificar y reflexionar sobre sus prácticas docentes, transformando las formas de enseñanza tradicional (De Pablos et al., 2016). Sin embargo, la implementación de estas metodologías requiere tiempo para la adaptación, formación pedagógica y reconocimiento por el trabajo realizado, lo que sin duda debiera ser considerado en la gestión universitaria.

\section{OTROS ANTECEDENTES}

Junto a las TIC, el trabajo colaborativo también ha evidenciado logros importantes en el proceso EA, al involucrar al estudiante en forma activa, mejorando sus habilidades mediante la cooperación e interacción. Una de estas estrategias colaborativas, es la metodología Aprendizaje Basado en Equipos (TBL, del inglés Team Based Learning), la cual se basa en la interacción de pequeños grupos y el reemplazo de la clase magistral tradicional por una actividad participativa. Este método se utiliza cada vez más en educación médica porque aborda competencias profesionales y mejora la experiencia de aprendizaje (Sweet y Michaelsen, 2007; Parmelee, 2010). El TBL comienza con el Proceso de Aprendizaje Inicial (RAP, del inglés Readiness Asurance Proceses), que consiste en asignar la lectura de un texto relacionado con el contenido y que el estudiante debe estudiar previamente. Durante la clase, la lectura es evaluada mediante una prueba de selección múltiple con un máximo de 10 preguntas. Primero en forma individual y luego, la misma prueba, en forma grupal para fomentar el análisis y discusión. En la segunda etapa, se utiliza un cartón de respuesta con marca oculta en la correcta, que al raspar, permite tener retroalimentación inmediata (Moraga y Soto, 2016). Posteriormente, el profesor revisa las respuestas con el curso para permitir apelaciones y argumentaciones válidas sobre respuestas erróneas; las que, si son lo suficientemente argumentadas, permiten mejorar la calificación. Este proceso beneficia el aprendizaje activo y colaborativo, al incorporar retroalimentación sobre los aciertos y fallas en las respuestas. EI RAP culmina con una clase expositiva corta y específica, que permite al profesor aclarar las dudas surgidas en las etapas anteriores. Finalmente, se emplean los conceptos de la unidad mediante un taller grupal de aplicación de contenidos. Generalmente los talleres se desarrollan con guías y con la participación de tutores que orientan y facilitan la discusión del grupo para responder colaborativamente.

\section{Otras metodologías}

Otro ejemplo de aprendizaje activo y colaborativo que es frecuentemente usado en la educación médica, es el estudio y resolución de casos (McLean, 2016). Consiste en el análisis crítico de problemas subyacentes, para identificar y suponer desafíos utilizando múltiples fuentes de datos o métodos múltiples con el propósito de avanzar en el conocimiento. El método, ya sea en forma grupal o individual, permite guiar la investigación de un fenómeno real - y que por lo general ha sido vivenciado por quien lo imparte - o utilizando un escenario clínico simulado con fines didácticos, y su esencia consiste en la búsqueda de una o más soluciones a una situación concreta. Un estudio de caso bien estructurado y claramente descrito, tiene el potencial de mejorar la comprensión de situaciones complejas a través de la participación activa del estudiante, el análisis crítico, la aclaración de los factores que contribuyen a la presentación de la situación concreta y el desarrollo de aprendizajes y habilidades gracias al protagonismo que adquieren los participantes (Cheek et al., 2018; Artiles et al., 2016).

Los entornos virtuales, también son ampliamente utilizados para trabajos colaborativos. Kahootß (se encuentra disponible en la página web con el mismo nombre) es una plataforma de aprendizaje, en línea y gratuita, basada en el juego y orientada al intercambio y colaboración mediante una aplicación que puede ser utilizada desde cualquier dispositivo móvil. Consiste en utilizar pruebas de selección múltiple y/o encuestas, con la opción de incorporar instrumentos multimedia como imágenes y videos entre otros, en donde los estudiantes responden en forma individual o en grupos pequeños, mediante el uso de sus teléfonos móviles, 
permitiendo la discusión grupal antes de responder. La aplicación permite que los estudiantes compitan (por respuestas correctas y tiempo), favoreciendo la interacción entre ellos y con el profesor, quien administra desde su computador las respuestas correctas y puntuaciones. Tiene la ventaja ya descrita de favorecer la retroalimentación inmediata acerca de las respuestas correctas y errores cometidos, que son aclarados por los propios pares y/o por el profesor.

La clase invertida es otra metodología, donde los estudiantes se hacen responsables de su propio proceso de aprendizaje. Consiste en que el profesor graba un video con la clase mediante plataformas virtuales y transforma la cátedra tradicional en una tarea, que el estudiante puede hacer en su hogar u otro lugar. Posteriormente, en la clase presencial, el profesor destina algunos minutos iniciales para discutir el video y resolver interrogantes. Finalmente, se realizan actividades de aplicación de contenidos tales como; resolución de problemas, desarrollo de casos, laboratorios, investigación o pruebas para la aplicación de los contenidos. La herramienta virtual gratuita más utilizada para la clase invertida, es el software Screencast-O-Matic $\circledast$ (se encuentra disponible en la página web con el mismo nombre), que permite al profesor grabar y editar una clase o tutorial utilizando pantalla y cámara web, mezclar presentaciones con diapositivas, archivos de audio, videos importados y publicar en cualquier medio en línea, como por ejemplo en una plataforma educativa institucional.

Considerando lo anterior, este trabajo se realizó en la Universidad Católica del Norte (UCN), institución que ha definido en su Proyecto Educativo Institucional 2017 (disponible en la página web de la UCN), que los ejes fundamentales del principio pedagógico son el desarrollo del aprendizaje esencialmente activo, el trabajo en equipo, trabajo autónomo y uso de TIC. En este documento, la Universidad define las competencias como el "conjunto de atributos personales que, movilizados, permiten y explican un desempeño exitoso en un ámbito profesional de realización específico, es decir, implica la integración de un saber hacer, saber ser y saber" (página 37), competencias que son incorporadas en los currículum de todas las carreras y programas. Para el desarrollo de tales competencias, el Proyecto Educativo da realce a las siguientes orientaciones de docencia; el autoaprendizaje, como la habilidad de tipo metacognitiva que permite descubrir oportunidades de aprendizaje, construir nuevos conocimientos y transferirlos a nuevas situaciones, lo que se caracteriza por ser constructivo, fomentar la curiosidad y auto disciplina; el aprendizaje autónomo, que implica un compromiso y responsabilidad consigo mismo y el entorno, y de forma controlada, el estudiante puede dirigir y evaluar su forma de aprendizaje y logro de metas; el trabajo en equipo, que permite la coordinación y cooperación de un grupo en torno a propósitos y objetivos comunes; y el aprendizaje activo y colaborativo, que implica la conexión entre lo nuevo que se aprende con experiencias previas, y hace del aprendizaje un proceso subjetivo que cada individuo modifica constantemente de acuerdo a sus experiencias. Esto se ve enriquecido con el trabajo colaborativo, que al compartir en equipos sus motivaciones, fortalezas y debilidades en torno a intereses y objetivos comunes, permiten su construcción de manera colectiva.

Consecuente con lo anterior, las competencias específicas establecidas para la carrera de medicina - que permiten resolver problemáticas de acción de la profesión - se sustentan en dos pilares; formación disciplinar y formación en salud pública, investigación y educación. Las competencias genéricas - transversales y aplicables en cualquier situación - tienen relación con la formación integral institucional. Finalmente, las TIC, son consideradas herramientas necesarias para el mundo globalizado, incorporándolas al quehacer docente y que requieren de profesores con habilidades para seleccionar, adaptar y crear nuevos recursos de acuerdo a su entorno, grupo de estudiantes y al acelerado cambio tecnológico.

\section{Asignatura Salud Ambiental y Ocupacional (SAO)}

La Carrera de Medicina fue creada en el año 2003, en la Sede de Coquimbo, por la necesidad y demanda de profesionales del área en las regiones de Coquimbo y Atacama. La malla curricular está conformada por tres ciclos; Ciclo de Ciencias Básicas de 4 semestres, Ciclo Clínico de 6 semestres, y finalmente el Ciclo de Internado con una duración de 4 semestres. En el año 2007, se realizó la primera modificación de la malla curricular, con la incorporación de la asignatura Salud Ambiental y Ocupacional (SAO) en el octavo semestre, con el objetivo de agregar una visión individual y colectiva de situaciones de salud que permitan reconocer en forma interdisciplinaria los factores determinantes ambientales y ocupacionales de la salud; manejar integralmente los problemas de salud, derivar adecuada y oportunamente; y aplicar medidas preventivas a este tipo de problemas.

El programa de la asignatura SAO se impartía durante el Ciclo Clínico y abarcaba tres módulos: salud ambiental, salud ocupacional y, emergencias y desastres. Los objetivos del programa tenían relación con explicar los procesos de control de los factores determinantes de la salud ambiental y ocupacional, de acuerdo a la estructura legal sanitaria en Chile, y analizar los principales elementos de la gestión integral del riesgo y manejo de crisis frente a emergencias y desastres. El programa estaba orientado al cumplimiento del perfil del egresado de la carrera de Medicina, en cuanto a identificar los problemas epidemiológicos de mayor 
importancia y aplicar las medidas adecuadas para evitar o disminuir los procesos que afectan al individuo, la familia y la sociedad, fundamentando su ejercicio profesional desde un enfoque biopsicosocial inspirado en el humanismo cristiano, con especial énfasis en la dignidad y respeto de la persona. La asignatura presentaba una carga total de 4 horas cronológicas directas semanales, consistentes en: clases, talleres con tutores, salidas a terreno y actividades autónomas con investigación bibliográfica, redacción y presentación de monografía.

Institucionalmente, se ha definido que todas las asignaturas de las carreras de la Facultad de Medicina, deben ser evaluadas por los estudiantes a través de un instrumento que mide la satisfacción con la formación recibida y el grado en que las expectativas han sido satisfechas. Se realiza a través de la "Encuesta de Evaluación de Docencia de las Asignaturas y el Coordinador" (en adelante encuesta), que es diseñada y aplicada por la Oficina de Educación Médica (OEM) de la Facultad de Medicina de la UCN - que es un organismo técnico y asesor pedagógico que apoya a las carreras de la salud - y que contiene varias preguntas agrupadas en siete dimensiones; metodología docente, pertinencia curricular, proceso de evaluación, aspectos administrativos, vínculo estudiante - profesor, ayudantías y coordinación de la asignatura. Desde que SAO se incorporó al currículum, las evaluaciones de satisfacción de los estudiantes, registraron baja aceptación en las dimensiones "pertinencia curricular" y "ayudantías"; la primera dimensión se refiere a los nexos entre los objetivos, contenidos y actividades de la asignatura, con otros cursos de la carrera y la visión que tienen los estudiantes en el futuro medio laboral; por otra parte, la segunda dimensión, busca determinar si la ayudantías cumplen con resolver dudas, facilitan el autoaprendizaje, apoyan los objetivos de cátedra y se realizan en el tiempo adecuado. Por lo anterior, se hizo necesario incorporar mejoras en las estrategias pedagógicas, a través de metodologías didácticas activas.

\section{Mejoras docentes}

En el año 2012, en el marco de un proyecto educativo centrado en el estudiante, con el apoyo del Centro de Innovación Metodológica y Tecnológica de la UCN (CIMET) - unidad que vela por mejorar permanentemente la calidad del currículum con asesoría, formación en docencia y acompañamiento a los académicos en el proceso EA - comenzó la implementación paulatina de metodologías didácticas activas en la institución, las que se han mantenido permanentemente durante los siguientes años. Algunos ejemplos son mencionados en el Manual de Metodologías Didácticas (año 2013), creado por la OEM. A saber: Aprendizaje basado en equipos (Team Based Learning o TBL), mapas conceptuales, análisis de casos, aprendizaje basado en problemas, aprendizaje basado en proyectos, portafolio, video, trabajo colaborativo de pequeño grupo, revisión bibliográfica, salidas de terreno, aprendizaje basado en casos, entre otros. Estas mejoras docentes, formaron parte de las estrategias de mejoramiento continuo que implementó el Departamento de Salud Pública de la Facultad de Medicina (del cual la asignatura SAO estaba adscrita), siguiendo los lineamientos institucionales de docencia y cuya planificación didáctica es entregada y dada a conocer a todos los estudiantes al inicio de cada semestre. En el curso SAO, se incorporaron técnicas didácticas nuevas, como aprendizaje colaborativo y uso de TIC. El objetivo de este estudio fue evaluar el cambio producido en el rendimiento académico y satisfacción de los estudiantes; en relación a las mejoras docentes incorporadas, consistentes en metodologías activas y TIC; en la enseñanza de salud ambiental y ocupacional en estudiantes de medicina del ciclo clínico.

\section{METODOLOGÍA}

La sección metodología consta de 4 subsecciones: en la primera se presentan las mejoras docentes, formas de evaluación, calificaciones y satisfacción de los estudiantes con la formación recibida; en la segunda sección se comenta el tipo de estudio, participantes, definición y medición de variables e instrumentos de recolección de la información; en la tercera, el resguardo de los principios éticos; $y$, finalmente, se describen las pruebas estadísticas y programas computacionales utilizados para el análisis de la información.

\section{Mejoras docentes, formas de evaluación y calificaciones}

Se analizan cuatro aspectos relacionados con las mejoras docentes: trabajo colaborativo y uso de tic, vinculación de contenidos con la práctica clínica, evaluación y calificaciones, satisfacción de la asignatura

\section{Trabajo colaborativo y uso de TIC}

En el año 2012, se incorporó el uso de la metodología TBL. Para la etapa RAP, mediante el recurso plataforma educativa institucional Educ@ versión 2.0, se entregaron con anticipación lecturas asignadas según los contenidos de la unidad correspondiente. La prueba individual tuvo una ponderación de $60 \%$ y la grupal (con tarjeta raspe), un $40 \%$ de la calificación. 
En el año 2014, con uso de la plataforma institucional Educ@, se iniciaron los talleres en línea y en tiempo real. Se utilizó el método de la clase invertida, con clases grabadas en el software Screencast - Matic®, se subieron al sistema las clases en videos y lecturas obligatorias. Se planificaron los talleres grupales de aplicación en fechas y horarios previamente acordados para facilitar la conexión en línea desde sus hogares o cualquier lugar con acceso a la red y que cada estudiante consideraba adecuado. Se permitió el trabajo en forma individual o grupal, a través de la sección "ejercicios" de plataforma Educ@ con preguntas de selección múltiple de respuestas únicas y múltiples. Las preguntas iban siendo liberadas por el profesor, quien monitoreaba el reporte de cada estudiante, tiempos de respuestas y registraba las calificaciones. Para permitir la discusión, el consenso entre pares antes de responder y la retroalimentación con el profesor, los estudiantes utilizaron la aplicación WhatsApp de sus teléfonos móviles, y para la aclaración y discusión con el profesor, se usó la sección "foros" de plataforma.

En los años 2015 y 2016, a raíz de las dificultades en las salidas a terreno por la ocurrencia de desastres naturales, se incorporó como trabajo autónomo el análisis crítico de videos guiados del sitio web YouTube®. Con guías de trabajo, los estudiantes diseñaron mapas conceptuales, en equipos de trabajo colaborativo, para exponerlos al curso presencialmente al final de la sesión. Desde el año 2015, comenzó el uso de la aplicación Kahoot $\AA^{\circ}$. Anticipadamente, se subió a plataforma material de lectura y/o audiovisual y guías de trabajo. Antes de cada taller, se desarrolló la clase interactiva con la entrega de un premio o incentivo a los 23 mejores equipos.

\section{Vinculación de contenidos con la práctica clínica}

Con el propósito de otorgar un enfoque y contexto médico, a partir del año 2013 se abordaron las áreas temáticas de determinantes ambientales y ocupacionales vinculándolos con aspectos clínicos. Por ejemplo; contaminación del aire y enfermedad respiratoria aguda-crónica, contaminación de alimentos y enfermedades transmitida por alimentos, contaminación por plaguicidas e intoxicación aguda-crónica, ambientes laborales contaminados y enfermedades ocupacionales tales como: silicosis, hipoacusia, patologías de origen toxicológico. Los elementos del módulo de emergencias y desastres, se entregaron con lecturas previas, clase participativa y un taller de simulación de situación de emergencia y/o desastre utilizando un caso real.

\section{Evaluación y calificaciones}

El rendimiento académico se midió a través de las calificaciones finales, que resultaron de los diversos métodos de evaluación utilizados. Se ha establecido que los estudiantes adaptan su aprendizaje a los métodos de evaluación, viéndose favorecidos cuando los docentes utilizan variedad de métodos, dado que los estudiantes presentan también diversidad de formas de aprendizaje (García-Prieto et al, 2019). La evaluación del aprendizaje de los estudiantes se realizó a través de pruebas teóricas de selección múltiple, TBL, guías y pautas de talleres, guía y pauta de evaluación de monografía, pauta de evaluación actitudinal de trabajo de grupo (promedios de autoevaluación, coevaluación y evaluación del tutor), guía y pauta de informes de terreno. Los instrumentos de evaluación (pruebas, TBL, pautas de talleres, pauta de evaluación de monografía, pauta de evaluación actitudinal) fueron similares en todo el período de estudio; incorporando cada año algunas variaciones y mejoras en los instrumentos, según los resultados del año anterior. Como fue explicado, con las mejoras docentes, se incorporó TBL (que debe ser evaluado), obligando a modificar las ponderaciones. Los métodos de evaluación del aprendizaje de los estudiantes y sus respectivas ponderaciones se presentan en la Tabla 1.

Tabla 1: Métodos de evaluación del aprendizaje y ponderaciones para la calificación final de la asignatura Salud Ambiental y Ocupacional. Carrera de Medicina Universidad Católica del Norte.

\begin{tabular}{|l|l|l|l|}
\hline $\begin{array}{l}\text { Método de Evaluación antes } \\
(2008-2011)\end{array}$ & $\begin{array}{l}\text { Ponderación } \\
\text { nota final }\end{array}$ & $\begin{array}{l}\text { Método de Evaluación después } \\
(2012-2016)\end{array}$ & $\begin{array}{l}\text { Ponderación } \\
\text { nota final }\end{array}$ \\
\hline Pruebas teóricas de alternativas & $45 \%$ & $\begin{array}{l}\text { Pruebas teóricas de alternativas } \\
\text { TBL }\end{array}$ & $\begin{array}{l}45 \% \\
5 \%\end{array}$ \\
\hline Talleres de trabajo en pequeño grupo & $10 \%$ & Talleres de trabajo en pequeño grupo & $15 \%$ \\
\hline Informe de Terreno & $20 \%$ & Informe de Terreno & $15 \%$ \\
\hline Monografía & $20 \%$ & Monografía & $15 \%$ \\
\hline Actitudinal de trabajo de grupo & $5 \%$ & Actitudinal de trabajo de grupo & $5 \%$ \\
\hline
\end{tabular}

\section{Satisfacción de la asignatura}

La evaluación de satisfacción de los estudiantes con las asignaturas se realiza habitualmente en la mitad y al finalizar cada semestre, a través de la encuesta de la OEM (se encuentra disponible en la página web de la OEM, UCN). La encuesta se aplicó en línea, fue de carácter anónima, con escala tipo Likert, fue la misma 
utilizada en todo el período de estudio y sólo al finalizar cada semestre. Este instrumento considera seis dimensiones: metodología docente, pertinencia curricular, proceso de evaluación, aspectos administrativos, vínculo alumno profesor y ayudantías. La escala va de 1 a 5, donde; 1 es nunca, 2 casi nunca, 3 a veces, 4 casi siempre y 5 siempre.

\section{Tipo de estudio, definición y medición de variables}

Se realizó un estudio de tipo cuasi experimental y retrospectivo, evaluando las mejoras docentes después de la introducción de metodologías didácticas activas (aprendizaje colaborativo) y uso de TIC. La evaluación de las mejoras se evidenció a través de dos variables: 1) el rendimiento académico, expresado en las calificaciones de los estudiantes y medidas de manera agrupada, a través de la mediana de las notas del curso respectivo de cada año; y 2) el nivel de satisfacción de los estudiantes con la asignatura, medido según la escala de la encuesta de evaluación de satisfacción con la asignatura, que se realiza normalmente al finalizar cada curso.

La muestra correspondió al total de 450 estudiantes de la carrera de Medicina de la UCN, que cursaron la asignatura SAO de manera sucesiva, en un periodo de nueve años (2008 al 2016). Se consideraron los años 2008-2011 para el periodo "antes" y años 2012-2016 para el período "después" de la introducción de mejoras docentes. La información de calificaciones, se obtuvo del sistema de registro institucional UCN (escala de 1 a 7 y calificación de aprobación 4,0 ; con un $60 \%$ de exigencia). Las notas finales de los 450 estudiantes fueron analizadas en ciego. Para determinar la satisfacción de los estudiantes, se utilizaron las observaciones de 343 encuestas de la OEM, disponibles desde el año 2009, por lo cual el grupo de estudiantes del año 2008 no fueron considerados; así como tampoco aquellos estudiantes que, en cualquier año, no la respondieron.

\section{Resguardo de principios éticos}

En principio, se debe contextualizar que las mejoras docentes estudiadas forman parte de la implementación del Proyecto Educativo Institucional. Siendo, además, recomendable sistematizar la implementación y evaluación de las nuevas estrategias pedagógicas para cumplir con el mandato académico de aportar al conocimiento; en este caso, en el ámbito de la educación médica. Tal como se describió, se utilizaron los registros de las evaluaciones habituales en la práctica docente, durante un periodo de 9 años; respetando la reglamentación institucional y los principios éticos de investigación. Las encuestas realizadas para evaluar las asignaturas fueron anónimas, resguardando así totalmente la confidencialidad de los estudiantes. EI registro de notas se analizó de manera ciega y agregada, como grupo curso, para cada año del periodo estudiado.

\section{Análisis de la información}

Los resultados de las calificaciones y encuestas de satisfacción de cada año, se ingresaron en una planilla Excel y luego se agruparon por períodos, antes (años 2008 a 2011) y después (años 2012 a 2016), de la introducción de mejoras docentes (metodologías activas y uso de TIC). Para determinar cambio en las calificaciones finales de los dos períodos; antes $(n=188$; y después $(n=262)$; se realizó análisis de regresión lineal simple con un $5 \%$ de nivel de confianza de significación. En la comparación de notas entre los dos periodos, se utilizó la prueba de T de Student para muestras independientes. Para relacionar las encuestas de satisfacción en ambos períodos, se calculó la diferencia porcentual de los puntajes y la significación estadística fue evaluada con la prueba $U$ de Mann-Whitney de mediana para dos muestras independientes (no paramétrica). Para el análisis estadístico se utilizó el paquete estadístico SPSS® V26.

\section{RESULTADOS}

Los resultados se presentan en 2 subsecciones: en la primera se describe el cambio en el rendimiento académico, medido a través de las calificaciones de los estudiantes antes y después de las mejoras; y en la segunda, se muestra la variación de la satisfacción de los estudiantes en ambos períodos.

\section{Calificaciones}

La tasa de aprobación promedio de los años 2008 al 2016 fue de 99,8\% y nota promedio 6,0 con una tendencia ascendente $(R=0,88 ; p=0,001)$. Situación similar ocurrió en la nota mínima $(R=0,83 ; p=0,004)$ y en menor medida en la nota máxima $(R=0,68 ; p=0,04)$. Como se presenta en la Figura 1 , el análisis del período pre incorporación de metodologías activas y TIC 2008-2011, demostró casi nula variación lineal, sin diferencias estadísticamente significativas en notas promedios, máximas y mínimas. En cambio, durante la etapa de mejoras docentes entre los años 2012-2016, se observó una fuerte dependencia funcional lineal en todas las notas y con cambios estadísticamente significativos $(p<0,002)$. 
En la Tabla 2 se presentan las calificaciones académicas promedios antes y después de la implementación de las mejoras docentes. Llama la atención que esta intervención coincide con mayores calificaciones de los estudiantes, aumentando significativamente, en especial las notas mínimas $(p=0,039)$ y en menor medida las notas máximas de los cursos.

Tabla 2: Calificaciones finales promedios antes (años 2008-2011) y después (2012-2016) de la incorporación de mejoras docentes, Salud Ambiental y Ocupacional (Prueba T Student).

\begin{tabular}{|l|l|l|l|l|}
\hline & Antes (2008-2011) & Después (2012-2016) & Diferencia & Valor de $p$ \\
\hline Nota promedio & $5,83 \pm 0,28$ & $6,12 \pm 0,29$ & 0,29 & 0,028 \\
\hline Nota máxima & 6,43 & 6,55 & 0,12 & 0,309 \\
\hline Nota mínima & 5,25 & 5,61 & 0,36 & 0,039 \\
\hline
\end{tabular}

\section{Satisfacción de los estudiantes}

La encuesta de satisfacción fue respondida por el $76,1 \%$ de los estudiantes que cursaron la asignatura (Tabla $3)$. En general se observa una mejoría en la satisfacción que fluctúa entre el $8 \%$ y $27 \%$. Todas las dimensiones de la encuesta subieron sus puntajes en el segundo período, siendo estadísticamente significativa en las dimensiones pertinencia curricular y ayudantías al comparar entre antes y después de implementar metodologías activas y colaborativas.

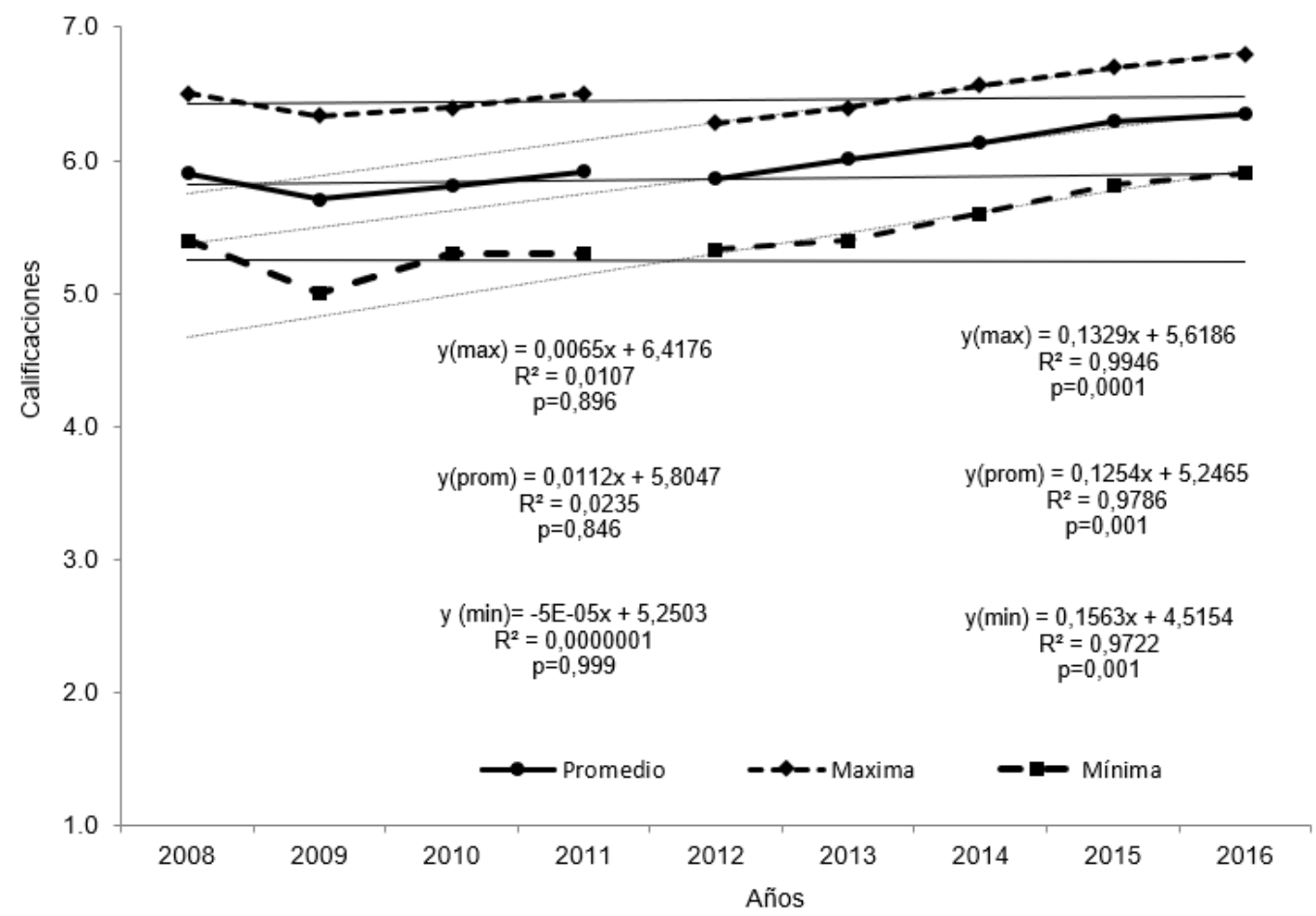

Figura 1: Regresión lineal de calificaciones finales antes (años 2008-2011) y después (2012-2016) de la incorporación de mejoras docentes, Salud Ambiental y Ocupacional.

Tabla 3: Satisfacción de estudiantes en promedios antes (años 2009-2011) y después (2012-2016) de la incorporación de mejoras docentes, Salud Ambiental y Ocupacional (Prueba U de Mann-Whitney de la mediana para 2 muestras independientes).

\begin{tabular}{|l|l|l|l|l|l|}
\hline Dimensión (Máx. 5 puntos) & $\begin{array}{l}\text { Antes } \\
(2008-2011)\end{array}$ & $\begin{array}{l}\text { Después } \\
(2012-2016)\end{array}$ & $\begin{array}{l}\text { Diferencia } \\
\text { absoluta }\end{array}$ & $\begin{array}{l}\text { Diferencia } \\
\text { porcentual }\end{array}$ & Valor de $p$ \\
\hline Metodología docente & 4,07 & 4,56 & 0,49 & $10,71 \%$ & 0,143 \\
\hline Pertinencia curricular & 3,89 & 4,47 & 0,58 & $13,08 \%$ & 0,036 \\
\hline Proceso evaluación & 4,13 & 4,67 & 0,55 & $11,79 \%$ & 0,071 \\
\hline Vínculo alumno profesor & 4,37 & 4,79 & 0,42 & $8,77 \%$ & 0,071 \\
\hline Ayudantías & 3,26 & 4,50 & 1,24 & $27,61 \%$ & 0,036 \\
\hline
\end{tabular}




\section{DISCUSIÓN}

Los resultados demostraron diferencias significativas en las calificaciones y satisfacción de los estudiantes después de la incorporación de mejoras docentes. De lo anterior se infiere que tales mejoras, en su conjunto, resultaron en ascenso, tanto de las calificaciones, como de la satisfacción de los estudiantes con la asignatura; descartando que estos resultados positivos sean debidos al azar. En cuanto a las calificaciones globales del curso, existió un incremento a partir de la agregación de metodologías activas y participativas, especialmente de las calificaciones promedio y mínima. Esto ha sido comprobado en estudios del área de docencia de la medicina, donde la dimensión pedagógica y habilidades de comunicación pueden influir en las calificaciones, e incluso ser independientes de la excelencia en el área de conocimiento y disciplina de los profesores (FloresHernández et al., 2016). Lo anterior podría explicarse por la optimización del aprendizaje producto de las nuevas intervenciones didácticas realizadas, y además por la participación de profesores con competencias y formación en docencia, producto del acompañamiento institucional en formación docente, metodologías didácticas activas y uso de TIC. Esto último resulta ser fundamental, considerando que en educación, se ha descrito que una de las bajas expectativas en el mejoramiento del proceso E-A, es la dificultad de algunas instituciones en incorporar y capacitar a profesores en TIC con sentido pedagógico, para lo cual se requieren cambios en la gestión estratégica, acceso a tecnologías, formación permanente de docentes y evaluación (Pino et al., 2016).

La significativa alza en las notas mínimas y promedios, como se señaló anteriormente, expresan que el cambio no fue debido al azar y que bien podría deberse a un aumento de los logros de aprendizaje y mayor comprensión de los contenidos de la asignatura, dada las intervenciones didácticas implementadas. La menor diferencia entre antes y después de las mejoras en la nota máxima, expresado en un menor aumento, se puede entender por la asíntota y el menor margen que existe para alcanzar la máxima calificación. Puede evidenciarse que la incorporación de las diversas metodologías docentes activas y colaborativas - no considerada en forma individual sino más bien en su conjunto, producto de la combinación de varias estrategias - repercutieron mayormente en mejorar las calificaciones de aquellos estudiantes que tuvieron menor rendimiento académico, lo que puede ser explicado por una mayor motivación hacia el estudio, facilitando la comprensión de conceptos, a través del aprendizaje vivencial y activo.

Una de las estrategias aplicadas permanentemente entre los años 2012 y 2016, fue el TBL. Se ha descrito que la metodología TBL mejora el rendimiento académico, facilita la adquisición de conocimientos, motiva al estudiante a ser activamente responsable de sus resultados de aprendizaje efectivo y fomenta el pensamiento crítico en carreras del área de la salud (Krase et al., 2018; Alwahab et al., 2018; Kazory y Zaidi, 2018). En este estudio, el incremento en las calificaciones finales pudo deberse a la incorporación de esta metodología, ya que debió fomentar la preparación, el estudio permanente, la retroalimentación inmediata y la vinculación con áreas de la medicina clínica, lo que coincide con los buenos resultados en tasas de aprobación reportados en cursos de pregrado de carreras de salud y ciencias básicas en Chile (Moraga y Soto, 2016).

El uso de clases invertidas, también pudo incidir en el mejoramiento de las calificaciones finales, principalmente porque este tipo de metodologías favorecen los niveles básicos del aprendizaje fuera del aula (conocimiento y comprensión) y permiten centrarse en las formas más elevadas de trabajo cognitivo en talleres presenciales (aplicación y análisis) (Zainuddin y Halil, 2018). Al respecto, existen evidencias del aumento significativo del rendimiento académico y satisfacción en estudiantes de las carreras de medicina, que han utilizado el método de aula invertida mediante videos interactivos con retroalimentación por el profesor, versus quienes reciben la enseñanza tradicional (Sezer y Abay, 2018).

Diversos autores reportan las ventajas del modelo del aula invertida, no obstante, aún se requieren más estudios que avalen si efectivamente produce actitudes más positivas, si el aprendizaje activo se atribuye a esta metodología y sobre todo, establecer evidencias de la utilización real del tiempo por los estudiantes (Akçayır y Akçayır, 2018). El uso de sistemas informáticos de gestión de cursos permite, de alguna manera, conocer esta evidencia, debido a que facilitan la retroalimentación para que el profesor descubra y monitoree si los estudiantes realmente se preparan antes de llegar a clase; además, permiten conocer qué les resulta difícil de entender en las lecturas previas y asignar calificaciones como incentivos para participar (Leasry et al., 2014).

En la actualidad, el empleo de TIC es ampliamente utilizado en la formación de estudiantes de medicina para la resolución de problemas, aprendizaje colaborativo y pensamiento crítico. También en el ámbito de la práctica médica, es reconocida su utilidad (Rodríguez-Padial et al., 2015; Cole et al., 2017). La utilización de Kahoot ${ }^{\circledR}$ como herramienta tecnológica, podría haber contribuido en el ascenso de las calificaciones y satisfacción de los estudiantes. Experiencias en docencia mediante la plataforma Kahoot® en estudiantes de medicina, han demostrado ser percibida como una herramienta de aprendizaje atractiva que promueve el aprendizaje activo, fomentando la participación debido a las recompensas que se entregan al finalizar. 
También es considerada como una fuente de motivación a través de la competencia entre pares, ya que aumenta la autoestima y estimula la participación, retroalimentación, auto-reflexión y autoevaluación, y en el ámbito del rendimiento académico, ayuda a mejorar la comprensión del tema abordado y preparar los exámenes (Ismail et al., 2019).

En el ámbito de la satisfacción con la formación recibida, las encuestas obtuvieron un puntaje significativamente superior en las dimensiones pertinencia curricular y ayudantías, después de la incorporación de metodologías activas y TIC, aumentando en un 13,08\% y $27,61 \%$ respectivamente. La deficiente evaluación de la pertinencia curricular que históricamente percibían los estudiantes, motivó a que se realizaran mejoras docentes en la asignatura a partir del año 2012 y porcentualmente obtuvo la segunda mayor diferencia. El ascenso obtenido en esta dimensión, se podría explicar debido a que las mejoras fueron desarrolladas junto con la inclusión de un enfoque y contexto del área médica clínica. A pesar de que las mejoras realizadas fueron esencialmente en metodologías docentes, la satisfacción de esta dimensión obtuvo menor alza que pertinencia curricular; probablemente debido a que la primera tenía inicialmente mayor puntaje. Respecto a los hallazgos en la dimensión ayudantías, su análisis debe estar incluido en el marco de talleres y trabajos grupales, pues era la instancia de aplicación de contenidos y conocimientos previos. La mayor diferencia encontrada entre antes y después, podría atribuirse a una mejor preparación de los talleres tomando en cuenta las estrategias autónomas utilizadas, como por ejemplo las clases invertidas; en tanto que la vinculación de los casos a la práctica médica clínica y el uso de TIC, favorecieron la integración, el trabajo activo y colaborativo.

La ejecución de metodologías colaborativas y el uso de TIC, coincidió con la mejor satisfacción en todos los ámbitos de la encuesta OEM con cambios estadísticamente significativos. Sin embargo, una limitación de este estudio, es que algunos factores que podrían haber afectado tal variabilidad, no pudieron ser excluidos; tales como las diferentes habilidades y competencias de los estudiantes que pueden estar presentes en las distintas promociones. Otra limitación de este estudio es que, al tener una orientación cuantitativa, reportó resultados numéricos de calificaciones y encuestas, sin explorar aspectos cualitativos que podrían emanar de opiniones de los estudiantes y que permitirían complementar, explicar e interpretar desde otra mirada los resultados de las mejoras docentes realizadas.

\section{CONCLUSIONES}

De los resultados de la investigación, de su análisis y discusión, se pueden obtener las siguientes conclusiones:

1) Las mejoras docentes implementadas en forma integral, con el uso planificado, adecuado y oportuno de metodologías activas de enseñanza - aprendizaje y la utilización de TIC, contribuyeron a fomentar el aprendizaje activo; traduciéndose en un aumento de la satisfacción con la asignatura y de las calificaciones en los estudiantes de la carrera de medicina, sobre todo en aquellos con menor desempeño académico.

2) El apoyo institucional, mediante el aseguramiento de la calidad y mejora continua, reflejado en el acompañamiento y compromiso con la formación docente, sin lugar a dudas, aportaron a estos resultados.

3) Los procesos de docencia universitaria y en particular en la educación médica, requieren incorporar el uso sistemático y permanente de metodologías activas y TIC, considerando sus beneficios y resguardos.

4) Se sugieren investigaciones futuras que incluyan metodologías cualitativas, para evaluar el efecto que tiene la incorporación de este tipo de estrategias en el proceso E-A.

\section{REFERENCIAS}

Akçayır, G., y Akçayır, M., The flipped classroom: A review of its advantages and challenges, https://doi.org/10.1016/j.compedu.2018.07.021, Computers and Education, 126, 334-345 (2018)

Alwahab, A., Abdulqader, S., y otros 5 autores, Team-based learning in an undergraduate pathology curriculum and its effects on student performance, https://doi.org/10.1016/j.jtumed.2018.03.010, Journal of Taibah University Medical Sciences, 13(5), 496-501 (2018)

Artiles, M.E., Artiles, C.J., y Rodríguez, F.E., El estudio de casos como método problémico en ciencias médicas: una experiencia necesaria, Edumecentro, 8(1), 165-173 (2016)

Bautista, G., Borges, F., y Forés, A., Didáctica universitaria en entornos virtuales de enseñanza - aprendizaje, 2ª̣ edición, 20-28. Narcea, Madrid, España (2008)

Bergmann, J., y Sams, A., Flip your classroom: Reach every student in every class every day, ISBN: 978-1-56484-315-9, International Society for Technology in Education - Association for Supervision and Curriculum Development, Washington DC. EEUU (2012) 
Castillo, M., Hawes, G., y otros 5 autores, Cambio educativo en las Facultades de Medicina, http://dx.doi.org/10.4067/S0034-98872014000800013, Revista Médica de Chile, 142, 1056-1060 (2014)

Cheek, C., Hays, R., Smith, J., y Allen, P., Improving case study research in medical education: a systematised review, https://doi.org/10.1111/medu.13469, Medical Education, 52(5), 480-487 (2018)

Cole, D., Rengasamy, E., y otros 4 autores, Using social media to support small group learning, https://doi.org/10.1186/s12909-017-1060-7, BMC Medical Education, 17(1), 1-7. (2017)

Coll, C., y Monereo, C., Psicología de la educación virtual, aprender y enseñar con las tecnologías de la información y comunicación, 19-123, ISBN: 978-84-7112-519-4, Morata S.L., Madrid, España (2008)

De Pablos, J., Colás, M.P., y González, T., La enseñanza universitaria apoyada en plataformas virtuales. Cambios en las prácticas docentes: el caso de la Universidad de Sevilla, Estudios Sobre Educación, 20, 23-48 (2016)

Flores-Hernández, F., Sánchez-Mendiola, M., y Martínez-González, A., Modelo de predicción del rendimiento académico de los estudiantes del ciclo básico de la carrera de medicina a partir de la evaluación del desempeño docente, Revista Mexicana de Investigación Educativa, 21(70), 975-991 (2016)

García-Prieto, F., Pozuelos-Estrada, F., y Álvarez-Álvarez, C., La evaluación de aprendizajes del alumnado por parte del profesorado universitario novel, http://dx.doi.org/10.4067/S0718-50062019000200003, Formación Universitaria, 12(2), 316 (2019)

Ismail, M.A., Ahmad, A., y otros 5 autores, Using Kahoot! as a formative assessment tool in medical education: a phenomenological study, https://doi.org/10.1186/s12909-019-1658-z, BMC Medical Education, 19(1), 1-8 (2019)

Kazory, A., y Zaidi, Z., Team-based learning activities for first-year medical students: perception of the learners, doi: 10.14423/SMJ.0000000000000865, Southern Medical Journal, 111(9), 525-529 (2018)

Krase, K., Pfeifer, E., y Swan, K., Team-based learning sessions compared with traditional lecture in the obstetrics and gynecology clerkship, doi: 10.1097/AOG.0000000000002856, Obstetrics \& Gynecology, 132(4), 14S-18S (2018)

Leasry, N., Dugdale, M., y Charles, E., Just in time to flip your classroom, https://doi.org/10.1119/1.4849151, The Physics Teacher, 52, 34 (2014)

McLean M, Cilliers, F., y Van Wyk, J.M., Faculty development: yesterday, today and tomorrow, https://doi.org/10.1080/01421590802109834, Medical Teacher, 30: 555-84 (2008)

McLean, S., Case-based learning and its application in medical and health-care fields: a review of worldwide literature, doi:10.4137/JMECD.S20377, Journal of Medical Education and Curricular Development, 3, 39-49 (2016)

Ministerio de Educación, Documento de trabajo $N^{\circ}$ 9. Una nueva tipología institucional de investigación, desarrollo e innovación $(1+D+i)$ en las universidades chilenas, Santiago, Chile (2017)

Moraga, D., y Soto, J., TBL-Aprendizaje Basado en Equipos, http://dx.doi.org/10.4067/S0718-07052016000200025, Estudios Pedagógicos XLII, 2, 437-447 (2016)

Organización de las Naciones Unidas para la Educación, la Ciencia y la Cultura., Directrices para los Recursos Educativos Abiertos (REA) en la educación superior, UNESCO, París, Francia (2015)

Parada, M., Romero, M.I., y Moraga, F., Perfiles de egreso de las carreras de medicina en Chile, http://dx.doi.org/10.4067/S0034-98872015000400014, Revista Médica de Chile, 143(4), 512-519 (2015)

Parmelee, D.X., Team-based learning: moving forward in curriculum innovation: $A$ commentary, https://doi.org/10.3109/01421590903548554, Medical Teacher, 32(2), 105-107 (2010)

Pérez C., Fasce, E., y otros 3 autores, Percepción de académicos de carreras de la salud de Chile sobre el perfeccionamiento docente, http://dx.doi.org/10.4067/S0034-98872013000600014, Revista Médica de Chile, 141(6), 787792 (2013)

Pinto A.R., Díaz, J., y Alfaro, C., Modelo espiral de competencias docentes TICTAPTEC aplicado al desarrollo de competencias digitales, Revista Educativa Hekademos, 19, año IX, 39-48 (2016)

Rodríguez-Padial, L., Cacheiro-González, M.L., y Medina-Rivilla, A., Conocimiento y uso de las tecnologías de la información y de la comunicación en la formación médica continuada, http://dx.doi.org/10.4321/S201498322015000500010, FEM: Revista de la Fundación Educación Médica, 18(4), 283-291 (2015)

Sezer, B., y Abay, E., Looking at the impact of the flipped classroom model in medical education, https://doi.org/10.1080/00313831.2018.1452292, Scandinavian Journal of Educational Research (2018)

Sweet, M., y Michaelsen, L.K., How group dynamics research can inform the theory and practice of postsecondary small group learning, https://doi.org/10.1007/s10648-006-9035-y, Educational Psychology Review, 19(1), 31-47 (2007)

Troncoso, D., Pérez, C., y otros 3 autores, ¿Se relaciona la capacitación docente con las prácticas pedagógicas en académicos de carreras de la salud de Chile?, http://dx.doi.org/10.4067/S0034-98872017000500008, Revista Médica de Chile, 145(5), 610-618 (2017)

Zainuddin, Z., y Halil, S.H., Flipped classroom research and trends from different fields of study, The International Review of Research in Open and Distributed Learning, 17(3), 1-17 (2018) 
